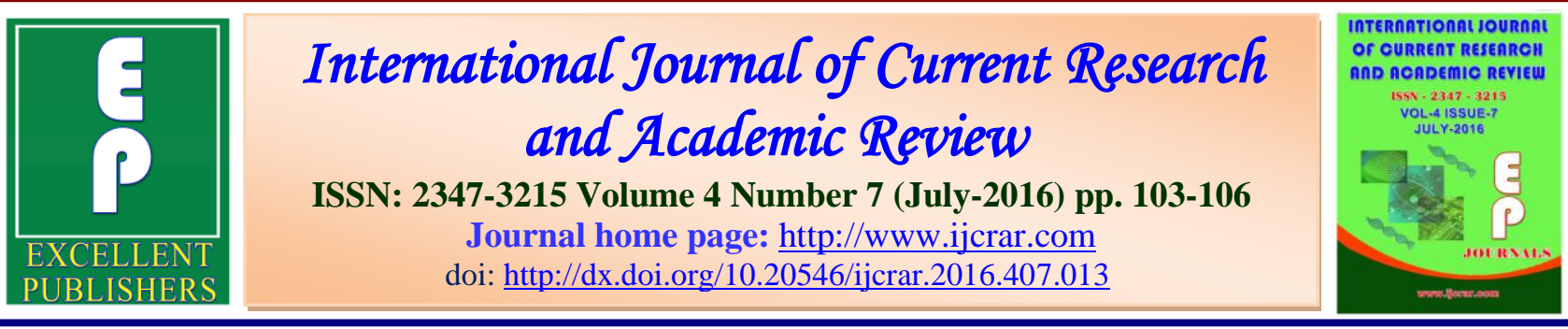

\title{
A Review on Quality of Life Assessment among Patients with Type 2 Diabetes Mellitus
}

\author{
Mathew George ${ }^{1}$, Lincy Joseph ${ }^{2}$ and Linu V. Koshy ${ }^{3 *}$ \\ ${ }^{1}$ Department of Pharmacology, Pushpagiri College of Pharmacy, Thiruvalla, Kerala, India \\ ${ }^{2}$ Department of Pharmaceutical Chemistry, Pushpagiri College of Pharmacy, Thiruvalla, \\ Kerala, India \\ ${ }^{3}$ Pushpagiri College of Pharmacy, Thiruvalla, Kerala, India \\ *Corresponding author
}

\begin{tabular}{l|l} 
KEYWORDS & A B S T R A C T \\
\cline { 2 - 2 } $\begin{array}{l}\text { Type 2 Diabetes } \\
\text { Mellitus, Quality of } \\
\text { life (QoL), } \\
\text { QOLID, }\end{array}$ & $\begin{array}{l}\text { Type 2 Diabetes Mellitus has a great impact in the quality of life of patients. } \\
\text { Quality of life should be assessed and routinely practiced in clinics thus helps } \\
\text { in achieving targets of diabetes management. }\end{array}$
\end{tabular}

\section{Introduction}

Diabetes mellitus (DM) is a heterogeneous group of metabolic disorders characterized by hyperglycemia. It is associated with abnormalities in carbohydrate, fat, and protein metabolism and may result in chronic complications including microvascular, macrovascular, and neuropathic disorders (DiPiro et al., 2014).

Diabetes mellitus is one of the major chronic non communicable diseases that affect millions globally. The number of people with diabetes has risen from 108 million in 1980 to 422 million in 2014. The global prevalence of diabetes among adults over 18 years of age has risen from $4.7 \%$ in 1980 to 8.5\% in 2014 (Mathers et al., 2006).
Quality of life can defined as "The state of contentment in a conscious individual due to his or her satisfaction in physiological, psychological, social and spiritual aspects of life" (Sithara).

According to WHO, " Quality of life is defined as individual's perceptions of their position in life in the context of the culture and value systems in which they live and in relation to their goals, expectations, standards and concerns (PubMed Nagpal)."

Quality of life evaluation has emerged as an important outcome measure for chronic disease management (Strom). It is increasingly recognized that in diabetes 
psychosocial factors have an important impact on self-care, acceptance of therapeutic regimens and treatment success. Metabolic measures like glycemic control are poorly correlated with quality of life necessitating separate assessment. In turn, management models for diabetes that include strategies to identify and enhance patient's health-related quality of life issues have the potential to improve compliance and hence their metabolic status. Quality of life is an individual perception and each particular subset of patients differ in their perception of quality of life influenced by their ethnicity, culture, education, income, etc (Strom).

The recognition of the patient-important (versus disease-oriented) and patient reported areas of well-being led to the introduction of a technical term: healthrelated quality-of-life (HRQL) (Strom).

As a result of changes in the lifestyle, food habits, and levels of physical activity the prevalence has increased in the past few decades. The fact that treatment for the disease and its associated risk factors are highly complex, a considerable patient education and medical monitoring are required.

Thus, the patients required to regulate the blood sugar level by making required changes in lifestyle factors and the unpleasant medication that usually accompanies the disease in order to maintain a correct degree of metabolic control. The fact that these changes make the patients vulnerable to stress, their quality of life is highly bound to be affected (Nyanzi et al., 2006).

The predictors of quality of life of diabetic patients are identified as personal, medical, and lifestyle factors.

\section{Literature Review}

Ronald Nyanzi et al., (2013).conducted a study on Diabetes and Quality of Life: A Ugandan Perspective

The predictors of quality of life in the dimensions of role limitation were patient's age, education level, $(P<0.05)$. Similar variables were associated with quality of life in the dimension of physical endurance $(P<$ $0.05)$.

The findings with regards to the influence of these variables can be summarized as follows.

(i) Quality of life was approximately $13 \%$ and $18 \%$ lower for diabetic patients above 59 years of age when compared to those below 50 years in the domains of role limitation and physical endurance, respectively.

(ii) Quality of life was about $16 \%$ and $19 \%$ higher in the domain of role limitation among patients with secondary and tertiary education, respectively, when compared to those with no education. Likewise, quality of life was about $11 \%$ and $16 \%$ higher among patients with secondary and those with tertiary education in the domain of physical endurance when compared to those with no education.

(iii) Quality of life of diabetic patients did not vary significantly by gender, status of smoking and alcohol consumption, treatment therapy, and type of diabetes as well as prevalence of hypertension and retinopathy $(P>0.05)$.

Ana Spasic et.al (2014) conducted a study on Qol in type2 diabetes patient. The presence of diabetes mellitus leads to a 
decrease in life quality in all domains. The aim of the study was to evaluate the quality of life (QoL) in diabetic patients and the factors affecting it in type 2 diabetic mellitus patients.

They conducted a cross-sectional study that included 86 patients with type 2 diabetes mellitus, in the territory of the City of Nis. Health-related QOL of patients was measured using the short form survey (SF36) that produces an 8-scale health profile.

The average duration of diabetes was $12.76 \pm 8.08$ years. The best QOL in all areas was observed in patients diagnosed with diabetes less than 10 years ago $p<0.05)$ and younger than 65 years. Male respondents perceived a better QOL compared to women, especially in the vitality and pain domains. The patients with comorbidity $(93.64 \%)$ had lower QOL score in all domains. There was no significant difference in the QOL of patients with diabetes compared to the level of education. High QOL represents an ultimate goal and an important outcome of all medical interventions in diabetic patients. Factors related to lower QOL included: older age, female gender, and existence of comorbidities. Uncontrolled diabetic patients had a lower QOL than controlled diabetics.

Harsimran Singh, Clare Bradley (2006) Conducted a study on Quality of life in diabetes as a key outcome of diabetes management and introduces the linguistically validated and culturally adapted, Hindi and Punjabi versions of an individualised questionnaire( the ADDQoL) to assess the impact of diabetes on the QoL of Indian people with diabetes. ADDQoL findings from research in India have helped highlight the negative impact of diabetes on various life domains of Indian people with diabetes, especially their self confidence, their family life and their freedom to eat as they wish.

It is suggested that the targets of diabetes management are more likely to be achieved if the importance of predicting and improving QoL is recognised and monitored alongside biomedical outcomes such as blood glucose levels.

Manjunath et al., (2014) conducted a study on Quality of life of a patient with Type 2 diabetes: A cross-sectional study in rural south India. With a high prevalence of diabetes in India, there is a need to study the impact of this disease on the quality of life (QoL) of the patients. This facility based cross- sectional study assessed the QoL of patients attending the diabetic clinic using the World Health Organization (WHO) QoL BREF instrument in Tamil Nadu. The QoL was analyzed domain-wise and various socio-demographic factors affecting the QoL were studied.

The mean total score of the QoL scale was 58.05 (95\% CI, 22.18-93.88). Domain-wise, $63 \%$ had good physical, 69\% had good psychological, $27 \%$ had good social and $85 \%$ had good environmental QoL scores. Males, currently married and those with BMI more than 25 had a statistically significantly better QoL compared to their counterparts.

Diabetes does impair the QoL of patients but not to a great extent. There is a need to specifically target and improve the QoL of women, widowed and separated, and nonobese diabetics who are at risk of a poor QoL. QoL assessment should be routinely practiced in diabetic clinics. 


\section{Conclusion}

Quality of life is an important tool for outcome measure for chronic disease management. The psychosocial factors have an important impact on self-care, acceptance of therapeutic regimens and treatment success.

Diabetes is a non-curable disease but can be controlled if effective steps were taken at right time thus preventing its progression and hence improves the quality of life. It is essential to assess the impact of diabetes on quality of life for improving diabetic care. High quality of life represents the ultimate goal and an important outcome of all medical interventions in diabetic patients.

The study concludes by giving emphasis on the fact that, quality of life assessment should be made into practice in clinics for improving diabetes care.

\section{References}

DiPiro, J., Pharmacotherapy: a pathophysiologic approach. New York; Madrid: McGraw-Hill Education. 9ed. 2014; 2294.

Diabetes Epidemic in Indians Cadi [Internet]. Cadiresearch.org. Available from: http://www.cadiresearch.org/topic/dia betes-indians.

Manjunath, K., Christopher, P., Gopichandran, V. et al. 2014. Quality of life of patient with type 2 diabetes:
A cross-sectional study in Rural South India. J. Fam. Med. Primary Care, 3(4): 397.

Mathers, C., Loncar, D. 2006. Projections of Global Mortality and Burden of Disease from 2002 to 2030. PLoS Med., 3(11): e442.

Nyanzi, R., Wamala, R., Atuhaire, L. 2014. Diabetes and Quality of Life: A Ugandan Perspective. J. Diabetes Res., 1-9.

Pub Med Nagpal, J. et al. The development of 'Quality of Life Instrument for Indian Diabetes patients (QOLID): a validation and reliability study in middle and higher income. Available from:http://www.ncbi.nlm.nih.gov/pub $\mathrm{med} / 21117348$

Singh, H., Bradley, C. 2006. Quality of life in diabetes. International Journal of Diabetes in Developing Countries. 26(1): 7.

Sithara, S.P. Validation of the diabetic foot ulcer scale short form (DFS-SF) and the measurement of health related quality of life (HRQL) among patients with type 2 diabetes in southern Kerala.Available from: http://dspace.sctimst.ac.in/jspui/bitstre am/123456789/2133/1/MPH_6150. pdf.

Spasic, A., Radovanovic, R., Dordevic, A., Stefanovic, N. et al. 2014. Quality of Life in Type 2 Diabetic Patients. Acta Facultatis Medicae Naissensis, 31(3).

Strom, B. 2005. Pharmacoepidemiol., 4ed. 653-4.

\section{How to cite this article:}

Mathew George, Lincy Joseph and Linu V. Koshy. 2016. A Review on Quality of Life Assessment among Patients with Type 2 Diabetes Mellitus. Int.J.Curr.Res.Aca.Rev.4(7): 103106. doi: http://dx.doi.org/10.20546/ijcrar.2016.407.013 